\title{
Téoros
}

Revue de recherche en tourisme

\section{0-1929 : affirmation du Québec comme destination touristique}

\section{Réjean Prévost}

Volume 14, numéro 2, été 1995

Le tourisme : toute une histoire!

URI : https://id.erudit.org/iderudit/1075095ar

DOI : https://doi.org/10.7202/1075095ar

Aller au sommaire du numéro

Éditeur(s)

Université du Québec à Montréal

ISSN

0712-8657 (imprimé)

1923-2705 (numérique)

Découvrir la revue

Citer cet article

Prévost, R. (1995). 1900-1929 : affirmation du Québec comme destination

touristique. Téoros, 14(2), 15-19. https://doi.org/10.7202/1075095ar d'utilisation que vous pouvez consulter en ligne.

https://apropos.erudit.org/fr/usagers/politique-dutilisation/ 


\section{0-1929: affirmation du Québec comme destination touristique}

\section{Réjean Prévost*}

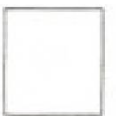

Il avait fait tout le jour ainsi que les précédents une chaleur excessive, et telle qu'on ne ressent que danis les pays les plus méridionaux de l'Europe: le frais de la soirée et la beauté du paysage, qui quoique sauvage présentait mille objets que la nature s'est diverti à former, m'invitèrent autant que les eatux chires de cette rivière à me baigner...(i)

C'est en ces termes que Louis Franquet, ingénieur du roi Louis XV et directeur général des fortifications en NouvelleFrance, s'exprima lors d'une tournée d'inspection dans la région de Trois-Rivières en 1752.

Sans pour autant jeter le discrédit sur le célèbre *quelques arpents de neiges de Voltaire, ce passage permet néanmoins à ce voyageur - qui, il faut bien en convenir, fait sans conteste partie des premiers touristes d'affaires au Québec - d'atténuer la simplicité des propos d'un individu qui $n$ 'a jamais daigné franchir l'Atlantique pour poser un regard *éclairés sur certaines caractéristiques que révélait alors au voyageur l'espace québécois.

Quelques décennies plus tard, une quantité croissante de voyageurs, majoritairement composée d'Américains et de Canadians viendront séjourner ou, à tout le moins, voir certains lieux de cette contrée qu'on appelait Bas-Canada. Ces visiteurs, qu'on a graduellement qualifié de touristes $^{(2)}$, c'est-à-dire, d'individus voyageant pour leur plaisir, vont notamment découvrir le Saint-Laurent et ses environs à bord de bateaux à vapeur. On verra alors se développer, dans certaines régions limitrophes au fleuve, une infrastructure hôtelière apte à répondre aux besoins de ces nouveaux résidents temporaires. Certains de ces visiteurs vont même en venir à établir leur résidence d'êté sur les rives du SaintLaurent.

Monseur Réjean Prévost, diplömét en tourisme et détenteur d'une maitrise en géographie de IUOAM est chercheur autonome.

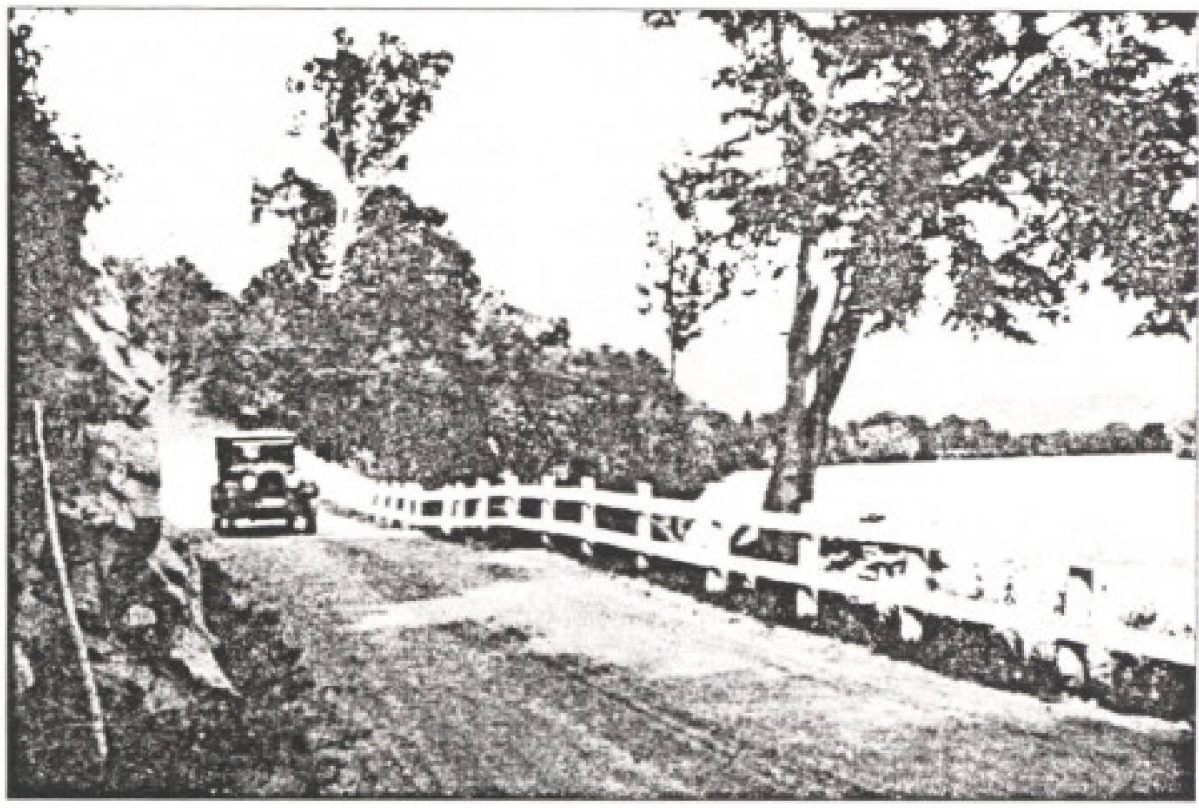

* Le réseau routier du Québec est l'un des plus populaires en Amériquew. Cette affirmation accompagnait la présente photographie, tirée de : Sur les routes de Québec, Guide du touriste, Ministère de la Voirie et des Mines, Québec, nowembre 1929 , p. 50.

L'avềnement du chemin de fer dans la deuxième moitié du XIX $\mathrm{XI}^{*}$ siècle aura comme effet d'étendre le phénomène ailleurs au Québec. Il se développera graduellement un engouement pour certaines autres régions, alors relativement confinées à l'isolement, comme les Laurentides et les Cantons de l'Est. Encore là, l'espace sera transformé pour accueillir lesvisiteurs. Comme le précise à ce sujet Marcel Samson:

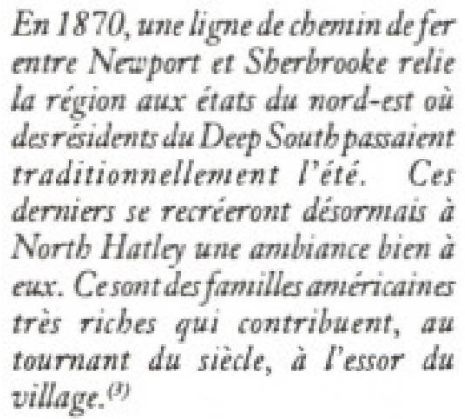

Cent cinquante ans après le voyage de Louis Franquet, alors que le mouvement d'appropriation du sol et de rentabilisation del'espace québécois à des fins touristiques est déjà enclenché, comment s'orchestrait l'activité touristique et quelles sont donc les particularités qui font du Québec un lieu à visiter?

Dans le présent article, nous tenterons de connaitre brièvement les principaux acteurs de développement et, par l'entremise d'écritscorrespondant à la période étudiée, nous efforcer de faire un pied de nez à Voltaire et montrer que plusieurs auteurs ont observé autre chose que quelques arpents de neige.

\section{Un développement touristique tributaire des compagnies maritimes et ferroviaires}

L'organisation et le développement du tourismeau Québeca d'abord et avant tout étél'affaire des transporteurs. Au début, ce sont les compagnies maritimes qui ont eu le mérite de bien comprendre le potentiel des cours d'eau - le Saint-La urent notamment - et de l'environnement qui les ceinture. Les compagnies de chemin de fer ont, par la suite, joué un rôle tout aussi 
important en ouvrant l'arrière-pays québécois.

Acteurs privilégiés au $\mathrm{XIX}^{c}$ siècle, c'est encore autour d'entreprises de ces secteurs d'activitésque gravite une importante partie du tourisme au début du vingtième siècle. Outre leurs rôles de transporteurs, les compagnies maritimes et ferroviaires ont aussi largement contribué au développement de l'infrastructure hôtelière québécoise ainsi qu'à la promotion du Québec comme destination. À cet effet, et dans le but évident de promouvoir leurs services, les compagnies maritimes et ferroviaires en sont venues à publier ou éditer denombreuxouvrages, brochuresouautres documents qui nous permettent d'en savoir plus sur ce que le Québec avait à offrir aux touristes à cette époque.

Ainsi, en 1905, la Richelieu \& Ontario Navigation Co., éditait un ouvräge intitulé Niagara to the Sea. Dans cette publication, on prétendait que le voyage qui était offert par la compagnie était athe finest inland water trip in the worlds. Plusieurs éléments fort évocateurs étaient alors présentés.

Every turn that we take in Quebec brings us face to face with some memorial of the past [...]. But contrasts are common in Quebec, for bere the old and new meet together as they meet nowbere else on the continent [...] the atmospbere at tbis locality - Tadoussac - is especially bracing; and sailing, rowing, and freb or salt water fishingare pastimes greatly enjoyed; the salt air from the Gulf of St. Latrence and the breezes from the Saguenay bills meet bere, and probably at no place in North Anerica can the denizens from beated localities find greater relief or lay in a larger amount of bealt b tban Tadoussac. [...] bere, above all otber places, the grandest works of mansink intoinsignifiance, and the very silence seens to do bomage as to a god. Calm and unbroken is the solitude of nature in this, ber temple [...] Good fishing is to be bad anyobere on tbe rivers and lakes of the gloriously diversified region around Lake St. Jobn, the bome of the land-locked salmon or ouananicbe. and large game-deer, bear, moose and the wapiti are to be found in season, witb capable guides to lead the bunter to their native fastnesser. nowbere in the world will the sportsman and the lover of the grand and beautiful in nature find better reward for this foil (4)

Dans la même veine, le Chemin de fer national du Canada a édité plusieurs ouvrages à l'intérieur duquel on traite du Québec et des spécificités qui font de cet espace un lieu à visiter. Les documents étant abondants, nous nous limiterons à exposer les propos compris à l'intérieur de 2 volumes en particulier.

En premier lieu, prenons connaissance de certains extraits du livre Québec, la doulce province de Jean-Charles Harvey, paru en 1925.

Québec est la plus ancienne, la plus originale et la plusvaste des provinces canadiennes. [...] La seule nature, par le grandiose de son oeuvre, sans considérer le glorieux et pittoresque passé qui enchantait Parkman, exalte ict l'imagination. Les accidents du terrain, l'étendue des borizons, lampleur des cours d'eau, la multiplicité des lass, tout dépasse la commune mesure, tout rappelle le souffle épique qui agita cette surface aux Lointains âges de la terre. [...] $A$ quarante milles de Montrial, à quelques arpents d'un des petits villages de la région, c'est la nature sawvage, lalternance infinie des bauteurs et des vallées, d'innombrables lacs, la forét et son mystère, la solitude, les délices de la chasse et de la pécbe, la liberté, l'mprévu, laventure, l'illusion grisante de remonter quelques siecles en arriere et d'être un courcur des bois, sac à dos, écorchépar les browssailles, brialé par le soleil, buvant aux sources, faisant cuire ses aliments à feu de brindilles, sans souci du temps et de la fatigue, sans loi ni maitre. (")

Dans un deuxième temps, Ernest Schenck, avec La grande aventure, en 1927, dresse un portrait montrant la diversité de l'espace québécois.

La plus ancienne province du Canada avec la Nouvelle-Écosse, la plus vaste, la deuxieme en popularion, la seule grande région d'Amérique à porter le nom de
Nouvelle-France, le Québec possède La variétéd'uncontinent. (...) Qwoi de phus différent de la Gaspésie (...) que la contrée pastorale du Lac SaintJean? Quoi de plus dissemblable que celle-ci et la région limitropbe du Saguenay, arrosée par le plus singulier affluent du SaintLaurent, la rivière de la mort? Que de contrastes entre les Éboulements et la Malbaie, sur la rive nord du Saint-Laurent, escarpíe, montagneuse, robuste, et Saint-7ean-PortJoli et la Riviere-du-Loup, sur La rive sud aux contours suaves? [.... ${ }^{\text {ol }}$

\section{Apparition de nouveaux acteurs}

Dans le premier quart du vingtième siècle, la question touristique commence à intéresser, outre les compagnies de transportet les hốteliers/restaurateurs qui sont dćà largement impliqués, certains individus ou entreprises quiverront dansce phénomène uneopportunité d'affaires à exploiter. C'est notamment le cas de plusieurs commerces dedétail tels Eaton, Holt Renfrew \& Co, et Birks, ou d'éditeurs privés comme, par exemple, U. Perreault \& $\mathrm{Cie}^{(7)}$. Alors que certains vont se limiter à annoncer dans les guides ou dépliants touristiques, d'autres simpliquent de façon beaucoup plus concrète. A tutue d'exemple, en 1921, la prestigieuse compagnie de vêtement Holt Renfrew \& Co.publia, conjointement avec la Canada Steamship Lines Ltd., un innéraire pour les touristes désireux de faire un voyage entre Montréal et le Saguenay. Ce circuit, préalablement destuné à un marché anglophone, mettait particulièrement l'emphase sur le pittoresque des lieux ainsi que sur les éléments se rapportant à l'histoire.

\section{L'implication de l'État}

Il faut attendre 1912, sous le gouvernement de Lomer Gouin, pour voir l'État discuter de tourisme. Et encore, ce n'est pas tant le tourisme que l'extension des routes qui fait l'objet de débats en chambre ${ }^{(8)}$.

Cet intérêt soudain de l'État origine d'un constat montrant l'insuffisance et à la médiocritéd des voies routières québécoisespar rapport à la popularité croissante de l'automobile. A cet effet, le tableau 1 nousdonne un aperçu de la progression du nombre d'automobiles au Québec au cours de cette période. Ainsi, alors qu'en 1907, on ne 
retrouvait que 254 automobiles enregistrées au Québec ${ }^{(9)}$, cenombre passeen 1920 à un peu plus de 41500 , pour, par la suite, quadrupler en 1930.

Pour combler cette lacune, le gouvernement du Québec met sur pied, dès 1912, un programme de construction d'un réseaude grandes routes. Àla fin de 1922, le Québec comptait 44 grandes routes pour 3100 milles de longueur totale.

Les efforts du gouvernement en ce qui concerne l'amélioration des voies carrossables va se continuer de sorte qu'au commencement de 1929 le réseau routier comprenait, entre autres, 50 routes de première classe, dont la longueur est de 4972 milles. ${ }^{(10)}$

Conséquemment à l'extension du réseau routier et face à un achalandage local et étranger toujours en croisssance (voir tableau 2), l'implication de l'État québécois en tourisme va véritablement commencer, en 1923, par la publication d'un bulletin sur les conditions routières au Québec.

Quelques temps après cette première véritable implication, le gouvernement du Québec va intervenir auprès des hôteliers pour qu'ils fassent la promotion d'une certaine *cuisine locales et, en 1926, suite à la production d'une première carte routière et d'une brochure à !'intention des touristes américains intitulée Québec, the French-Canadian Province, se sera la parution par le ministère de la Voirie de l'ouvrage Voyez Québec d'abord, See Quebec First.

Ayant pour sous-titre Tours de fin de semaine et itinéraires de vacances suggérés aux automobilistes, ce document avaitd'abordcomme objectifd'inciter les Québécois à visiter les attraits de leur pays d'abord. À cet effet, un des passages de l'avant-propos évoque bien cette ambition.

Ce titre indigue assez clairement le but de la brocbure, qui est d'induire nos automobilistes à visiter et à connaittre leur province d'abord et avant tout. Au moment où la province de Québec est en voie de devenir la meeque des touristes de l'Amérique du Nord, il serait pour le moins étrange que nos automobilistes la delaissent pour aller voir ailleurs des sites ni plus intéressants que ceux de leur propre

Tableau 1

Automobiles enregistrées au Québec et au Canada pour la période 1920-1930

\begin{tabular}{lcc}
\hline Années & Québec & Canada \\
\hline $1920 \ldots .$. & 41562 & 408790 \\
$1925 \ldots$ & 97418 & 724048 \\
$1930_{\text {a.. }}$ & 178548 & 1232489
\end{tabular}

Source: Burcau fédéral de la statistique, Canada 1940, Manuel officiel des conditions présentes et des progrès récents, J.O. Patenaude Imprimeur, Ottawa, 1940, p. 135.

\begin{tabular}{cc}
\multicolumn{2}{c}{$\begin{array}{c}\text { Tableau 2 } \\
\text { Nombre d'automobiles de tourisme ayant traverse } \\
\text { la frontière de 1917 à 1928 }\end{array}$} \\
\hline Annces & Automobiles \\
\hline 1917 & 7427 \\
1918 & 9177 \\
1919 & 18105 \\
1920 & 31918 \\
1921 & 41957 \\
1922 & 95163 \\
1923 & 130099 \\
1924 & 199008 \\
1925 & 259959 \\
1926 & 265848 \\
1927 & 417561 \\
1928 & 500000 \\
$1929^{\circ}$ & 625000 \\
\hline
\end{tabular}

*Il s'agit d'une approximation.

Source : Ministère de la Voirie et des Mines, Sur les routes de Québec, Guide du touriste, Québec, novembre 1929 , p. 49.

pays. Sans vouloir amoindrir en rien les attraits que peuvent offrir les provinces et les états voisins, nous affirmons sans crainte de nous tromper que l'on n'y trouvera pas plus pittoresgue que dans notre province, at que nul part ailleurs Ton ne trauvera le quart de l'intérêt bistorigue qui s'attacbe à chaque coin de terre du vieux Québec.(an)

En 1927, pour faire suite à cette publication majeure et afin de coordonner ses efforts de promotion, le gouvernement du Québec créait sous la gouverne du ministère de la Voirie, le Bureau provincial du tourisme. Plusieurs documents y seront publiés dont un en 1929 intitulé $\mathbf{L a}$ Gaspésie, à l'intérieur duquel on peut lire: *Accompli avec l'aide de ce guide [...], le "tour de la Gaspésie", un circuit de 553 milles, constutuera la plus agréable, la plus instructive et la plus poétique des promenades $w^{(12)}$.
En novembre 1929, sortait Sur les routes du Québec, un volumineux document de 874 pages. En plus de suggérer différents circuits aux touristes, ce document permettait au lecteur d'en savoir un peu plus sur ce que le Québec avait à lui offrir. Par exemple, on y affirmait:

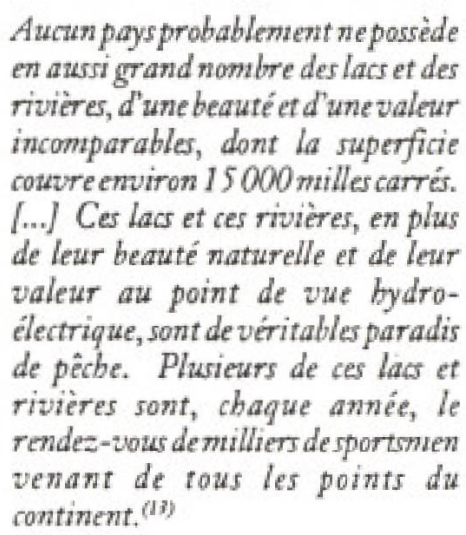

Au début des années 1930, plusieurs autres documents promotionnels seront également mis à la disposition des voyageurs. 
Mentionnons entre autres une brochure şur les Laurentides ${ }^{(14)}$, sur les Cantons de $l^{\prime} E s t^{(15)}$, et une autre sur le Lac SaintJean ${ }^{(16)}$.

\section{Des éléments de compara ison avec ailleurs}

Plusieurs documents-guides, récits et brochures, rédigés entre 1900 et 1929 et émanant de promoteurs, de reporteurs ou d'organismes gouvernementaux, en sont venus à exposer les attraits du Québec par analogie à un autre coin du globe ou tout simplement par un rapprochement établi en fonction de caractéristiques spatiales, sociales ou culturelles.

Parmi ceux qui ont apporté des éléments de comparaison dans leurs écrits, citons premièrement les propos de l'abbé V.-A. Huart. Ce dernier, lors d'un voyage qu'il effectuait en Europe en 1900, en est venu à étáblir une comparaison entre le Saguenay et le Rhin.

J'étais curieutu de faire connaissance avec le Rbin, que j"ai quelquefois entendu comparer avec notre rivière Saguenay. Eb bien, soit! Quel'on compare à ce flewve notre riviere, si parva licet componere magnis. D'abord, pour le valume des eatux, il $n$ 'y a pas entre eur de comparaison possible. Meme à Cologne [...], le Rhin est encore de moitté moins large que le Saguenay en face de Chicoutimi. Au milieu du parcoutrs $[\ldots]$, les rives du grand fleuve allemand deviennent assez pittoresques. Elles atteignent une bauteur de plusicurs centaines de pieds, s'élevant en pentes plus out moins abruptes, et presque partout admirablentent boisées. Mais qu'il y a loin de l'aspect agréable, assurement, qu'offre alors le Rbin, a Lasauvage et majestueuse grandeur que présente, sur presque tout son parcouts, notre sombre Saguenay [...]. En résumé, comme oenvre grandiose de la nature, le Saguenay est cent fois supérieur au Rhin, qui, de son côté l"mporte facilement sur celui-là comme oewvre naturelle embellie par la main des bommes. ${ }^{(17)}$

Jean-Charles Harvey, dans son ouvrage Québec, la doulce province, fait aussi le rapprochement de certains espaces territoriaux du Québec avec des éléments euro-

péens. Dans une première comparaison, l'auteur écrit en parlant des Cantons de l'Est: «Quelle sensation étrange de visiter, en paroourant la campagne, et dans un rayon de quelques milles, un coin de France, un coin d'Angleterre ou un coin d'Écosse $w^{\{1 / 1)}$

Dans un deuxième temps, l'auteur utilise une approche foncièrement descriptive pour présenter la Gaspésie et la comparer.

\begin{abstract}
$A b$ ! le charme des petits villages silencieux, de leurs maisonnettes blanches, écbelonnées en bordure de la route, sur le rivage de la mer, près du clocher de lighlise, pareilles aux brebis broutant sous la protection $d u$ berger, le ebarme des lumieres menues, toutes frissonnantes dans le vent du soir qui souffle du large, des champs de patates, de sarrasin ou d'avoine, aultives dans les intervalles de la pếche, de la prairie dont on coupe le foin vers la fin d'aồt, du quai primitif rongé par la lame, des filets mis à sécher sur la grève, des embarcations des pêcbeurs de morue, cbaloupes à voile ou canots autonobiles, du troupeau dont les clocbettes résonnent dans la montagne proche! Cette contrée colorée rappelle le littoral breton, et c'est encore la province de Québec.(19)
\end{abstract}

L'espace gaspésien a également été comparé dans certains autres documents. Ainsi, en 1927 dans un ouvrage édité par le Chemin de fer National, Ernest Schenck écrivait ce qui suit: *Quoi de plus différent que la Gaspésie, Bretogne par le littoral, Acadie par une partie de sa population, petite Terre-Neuve par la pêcheà la morue [...] $w^{(20)}$

La Richelieu and Ontario Navigation Co. se sert également d'une comparaison pour décrire un autre coin duQuébec soit Murray Bay et l'espace qui l'environne. *Murray Bay, with the grand old Laurentian mountains behind it and the river in front, furnished a variety of scenery not often found in combination. it has often been compared to the west coast of Scotland for Beauty, (ail)

De mème, dans le guide publié par le minisțteredela Voirie en 1930 etintitulé La Gaspésie, on évoque la similarité qu'il semble exister entrece territoire et I'Trlande. Il est notamment écrit à ce sujet:
Les montagnes et les vallées, les forêts et les lace de ce coin merveilleux du Canada reproduisent, avec une étonnante exactitude, les paysages Lesplus grandioses et les plus typiques de la vieille Irlande [...] Ce que fut I'Irlande dans les siécles passés, la Gaspésie l'est aujourd'bui. (2a)

Ce document ne se limite toutefois pas à cette seule comparaison puisqu'il note, pour caractériser le paysagesitué entre les villages de New Carlisle et New Richmond: *La route s'éloigne lentement, en courbe gracieuse, du bord de l'eau, et des paysages d'une délicieuse beauté s'offrent au regard du voyageur. C'est un autre coin de la Suisse, un splendide mélange de forêts, de montagnes, de vallées et de rivières $w^{(2)}$

Finalement, et même si le propos de JeanCharles Harvey sur la ville de Québec ne fait aucun rapprochement avec un lieu comparatifbien déterminé, le sensprofond des lignes qu'il consacre à cette entité géographique n'est toutefois pas dénudé de tout rapprochement lorsqu'il écrit:
L'étranger qui, tout à coup et sans voir les distances, se trowverait transporté à Québec, par un jour d'été, refuserait àsecroire dans une ville d'Amérique. Le cacbet vétuste que revêt les vieilles rues étroites et le caprice des toits inégaux, les lucarnes pointues, les modéles d'arcbitecture d'un autre äge, la pierre des fortifications, brunie par le temps, l'inégalité du plan d'ensemble de la cité, qui indigue diverses étapes bistoriques, tout cela, vu dans une homière intense conme la lumière méridionale, lui donnerait l'impression des plas aimalles contrées du vientronde, ${ }^{(4)}$

\section{Et puis...}

Il $y$ a l'hiver. Peu s'y sont attardés lors de certe période. Ne pas en parler serait en quelque sorte donner raison à Voltaire. Bien soit! Citons les propos d'un auteur :

While Quebec is preeminently a sumimer resort, it also claims the right to be crouned as Queen or Nortbern Winter Resorts, and it reserves some of its cbief attractions for its Winter visitors. Then, when the frost king is supreme, the population devotes a gencrousportion 
of its timue to the various forms of out-door pleasure and Winter sport which bave made St. Petersburgb ane of the most famous and popular European Winter Resorts. the time is spent in sleighing, skating, tobogganing, sking and snowshoeing, and such games as curling and bockey [...] Quebec, in Winter, is strongly recommended by eminent medical men as a resort for those predisposed to pulmonary complaints...(2)

Somme toute, l'intérêt que tant d'acteurs et de visiteurs ont démontré pour ce pays de quelques arpents de neige, semble, a priori, donner raison aux conquérants qui $y$ avaient vu beaucoup plus qu'une simple cabane au Canada. $f$

NOTES

11) Louis franquet, Voyages et mémoires sur le Canada 1752, Imprimerie A. Cóté et Cre, Quebec. 1889. p. 48 .

(2) Employé pour la premiere fois au debut du XIXsiede, le vocable touriste tife son ongine du w Grand Tours, un circuit doctutif pratique pour de jeunes nobles britanniques comme complement al leur formation.

(3) Marcel Samson, La route des viliegioteurs, in Continulte, numero 40 ete $1989, \mathrm{p} .14$

(4) Richelieu \& Ontario Navigation Co. Niagara to the Sea Official Guide, Montreal, 1905, pp. 52-54-7071 et 74

(5) Jean-Charles Harvey La doulce province tdis parle Chemin de ler National, Montréal, 1925, pp. 5 et 23 .

(5) Ernest Schenck, La grande aventure, edite par le Chemin de fer Natoonal du Canada, Montreal. 1927 D. 38

(7) En 1923, soulignons la parution d'un premier guide de 444 pages publié par les éditeurs U. Perreault et Cie el intitule Guide des touristes de la province de Ouébec. Cette initiative sera su: vie en 1924 par la parution du premier guide de coute par la Canadan Automoble Assoca

V Noustenons dremerciermonseur Gilles Gellich. conservateur-archiviste à la bibliotheque de I'Assemblée nationale du Québec, pour son assis tance tort précieuse lors de la recherche concernant les débats sur le tourisme

(9) Ministere de la Voirie, Voyez Qubbec d'abord, See Quebec Firat, Quebec, mai 1926. D. 48

(10) lbid.

(11) lbid. pp. 5 et 6

(12) Ministere de la Vorie, La Gaspósie Québec, jullet 1930 , p. 7

(13) Ministerede la Voirie et des Mines, Sur les routes de Oubbec, Guide du touriste, novembre 1929 pp. 21 et 28

[14] Voir a cet effet rotfice provincial du tourisme Laurentian Tours. Quebesc, 1932, 32 pages.

(15) Voir a cet effet l'Office provincial du tounisme, the Eastern Townships of Quebec, Quebec, 1932 12 pages

[16) Voir a cet eftet rolfice provincial du tourisme. Lake St. John and National Park, Québec, 1932. 12 pages.

(17) L'abbé V.A. Huart, Impressions d'un passant typ. Dussault \& Proulx, Quebec, 1906, p. 114

19. Jean-Charles Harvey, op. cit., p. 27

(19) bid..p. 35 .

20) Ernest Schenck, op. clt. p. 36.

21) Pichelieu \& Ontario Navigation Co. op. cit., P. 65.

122) Ministère de la Voirie, La Gaspésie, op. cit. p. 10.

(23) lbid. P. 218 .

(24) Jean-Charles Harvey, op. eit, p. 39

(25) Aichelieu \& Ontario Navigation Co, op, cit., pp. 55 56

\section{Les 100 ans du Château Frontenac \\ Marie-Janou Lusignan}

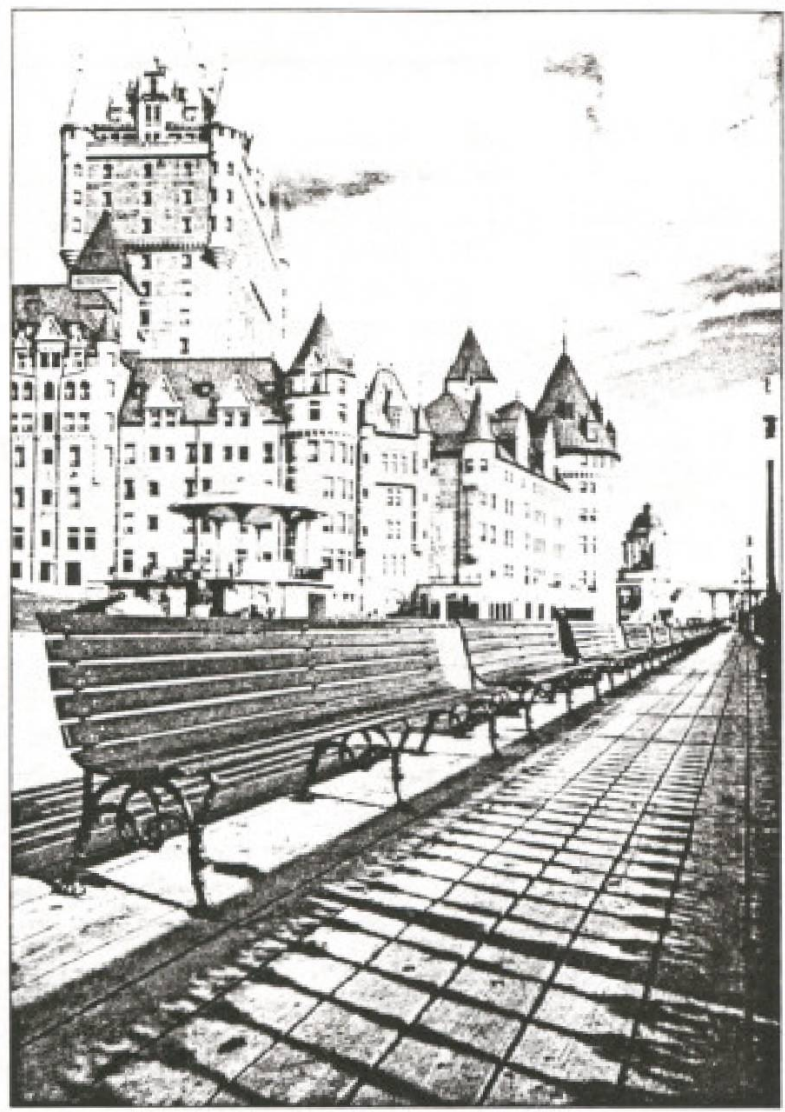

Le 20 décembre 1893, la première aile complétét du Chăteau Frontenac, l'aile Riverview, fut inaugurée dans le fastè qui convenait. Elle comprenait 170 chambres, dont 93 étaient pourvues d'unesalle debain etd'une cheminée, luxe inoui pour l'époque. L'hôtel comprenait aussi trois suites magnifiques.

Plusieurs agrandissements se sont succédés jusqu'en 1993 pour construire l'hôtel tel qu'il apparaît aujourd'hui. L'aile Citadelle a été construite en 1899 , suiviedel'aile MontCarmel en 1908, de l'aile Saint-Louis et de la Tour centrale construites entre 1920 et 1924 et dont l'allure rappelle les donjons des cháteaux de l'époque médiévale en France. Une nouvelle phase d'agrandissements a été complétée en juin 1993 avec l'aile Claude-Pratte.

Le Château Frontenac tient son nom du gouverneur français, Louis de Buade, Compte de Frontenac, qui dirigea les

Le Château Frontenac, haut lieu de romantisme et joyau d'architecture nord-américaine, célébrait en 1993 ses premiers 100 ans.

Après les Châteaux Saint-Louis et Haldimand qui ont hébergé les gouverneurs français et anglais durant plus de deux siècles, le promontoire du Cap Diamant avait perdu deson prestige et les projets d'un grand hôtel à Québec se succédaient sans jamais se concrétiser.

A la fin du siècle dernier, William Van Horne, alors président du Canadien Pacifique, réussit à réunir les fonds nécessaires à la réalisation du Chăteau Frontenac, qui serait une halte parfaite pour les voyageurs des trains de la compagnie Canadian Pacific Railways.

Van Horne retint les services de l'architecte new-yorkais Bruce Price, délà auteur de la Gare Windsor à Montréal. C'est à même les styles architecturaux du Moyen-Age et de la Renaissance que Price s'est inspiré pour immortaliser l'histoire des deux grandes puissances qui ont occupé le plus haut cap de Québec. destinées de la Nouvelle-France de 1672 a 1698. On trouve d'ailleurs ses armoiries sur la façade extérieure de l'arche d'entrée du Château, ainsi qu'à bien des endroits à l'intérieur de l'hôtel.

L'histoire est omniprésente à travers l'architecture du Chäteau Frontenac. Unepierre vieille de 300 ans et portant l'emblème de la Croix de Malte est placée parmi les pierres de l'intéricur de la voûte d'entrée de l'hötel.

Le Château Frontenac a aussi été l'hôte de nombreux evenements historiques notamment des Conférences de Québec qui réunirent, en août 1943 et septembre 1944 , durant la Deuxième Guerre mondiale, le Président américain Franklin D. Roosevelt, le Premier Ministre britannique Winston Churchill et le Premier Ministre canadien Lyon MacKenzic King.

Aujourd'hui, le Château Frontenac s'élève fièrement sur le Cap Diamant, à l'aube d'un nouveau siècle et dun nouveau millénaire.

Ce texte est le résumé d'un document trangms par madame Caroline Samson, directnce des relations publiques au Chătesu Frontenac

Photographie : Parcs Canada - Services photographiques. Photo : Louis Jacob 